\title{
Probabilités \\ Schémas d'approximation associés à une équation différentielle dirigée par une fonction höldérienne; cas du mouvement brownien fractionnaire
}

\author{
Ivan Nourdin \\ Université Henri Poincaré, Institut de Mathématiques Élie Cartan, B.P. 239 \\ 54506 Vandouvre-lès-Nancy Cédex, France \\ nourdin@iecn.u-nancy.fr
}

Résumé. Nous étudions les schémas d'approximation classiques (Euler, Milshtein) associés à une équation différentielle du type $d x_{t}=\sigma\left(x_{t}\right) d g_{t}+b\left(x_{t}\right) d t, x_{t} \in \mathbb{R}$, où $g$ est une fonction supposée höldérienne d'ordre $\alpha$ quelconque dans $(0,1]$. Quand $g=B^{H}$ est la trajectoire d'un mouvement brownien fractionnaire, nous tirons parti de propriétés probabilistes pour affiner les résultats.

\section{Cas d'une fonction $g$ quelconque}

1) Soit $g:[0,1] \rightarrow \mathbb{R}$ une fonction höldérienne d'indice $\alpha \in(0,1]$. Quand $\alpha \leq 1 / 2$, on ne peut pas utiliser une intégrale de type Stieltjes (considérée par Young [1]) pour intégrer contre $g$. L'intégrale de Newton-Côtes définie dans [2] permettrait de traiter le cas de tous les indices $\alpha$ mais le plus simple pour cette Note est d'introduire la:

Définition 1 Soit $f: \mathbb{R}^{2} \rightarrow \mathbb{R}$ de classe $\mathrm{C}^{1,1}$ et $a:[0,1] \rightarrow \mathbb{R}$ une fonction à variation bornée. On pose, pour $t \in[0,1]$,

$$
\int_{0}^{t} \partial_{1} f\left(g_{s}, a_{s}\right) \circ d g_{s}:=f\left(g_{t}, a_{t}\right)-f\left(g_{0}, a_{0}\right)-\int_{0}^{t} \partial_{2} f\left(g_{s}, a_{s}\right) d a_{s},
$$

l'intégrale contre a étant au sens de Stieltjes.

Soit $x_{0}$ un réel et $\sigma, b: \mathbb{R} \rightarrow \mathbb{R}$ deux fonctions régulières. On peut maintenant donner un sens à l'équation formelle

$$
\left\{\begin{array}{l}
d x_{t}=\sigma\left(x_{t}\right) d g_{t}+b\left(x_{t}\right) d t, t \in[0,1] \\
x(0)=x_{0}
\end{array}\right.
$$

Définition 2 Une fonction $x:[0,1] \rightarrow \mathbb{R}$ est solution de (2) si:

a) il existe $f: \mathbb{R}^{2} \rightarrow \mathbb{R}$ de classe $\mathrm{C}^{1,1}$ et $a:[0,1] \rightarrow \mathbb{R}$ à variation bornée tels que, pour tout $t \in[0,1], x_{t}=f\left(g_{t}, a_{t}\right)$;

b) pour tout $t \in[0,1]$, on a $x_{t}=x_{0}+\int_{0}^{t} \sigma\left(x_{s}\right) \circ d g_{s}+\int_{0}^{t} b\left(x_{s}\right) d s$.

Quand $\sigma$ est de classe $\mathrm{C}^{2}$ avec ses dérivées première et seconde bornées et quand $b$ est lipschitzienne, l'existence d'une solution de (2) est assurée par la méthode de Doss [3] et Sussmann [4]: posons $x_{t}=u\left(g_{t}, y_{t}\right)$ où $u: \mathbb{R}^{2} \rightarrow \mathbb{R}$ est (l'unique) solution de $\partial_{1} u=\sigma(u)$ 
avec $u(0, z)=z$ pour tout $z \in \mathbb{R}$ et où $y:[0,1] \rightarrow \mathbb{R}$ est (l'unique) solution de $\frac{d y_{t}}{d t}=$ $\left[\partial_{2} u\left(g_{t}, y_{t}\right)\right]^{-1} b\left(u\left(g_{t}, y_{t}\right)\right)$ avec $y_{0}=x_{0}$ (remarquons que $\partial_{2} u$ ne s'annule jamais car c'est une exponentielle: voir [5] p.296). On a noté $\partial_{1}$ (resp. $\left.\partial_{2}\right)$ la dérivée par rapport à la première (resp. deuxième) variable. On voit immédiatement, en utilisant (1), que $x$ ainsi construite est solution de (2). Pour l'unicité, nous renvoyons à [2], Théorème 4.3.3 p.82.

Dans la suite, nous considérerons comme solution de (2) la fonction $x$ donnée par la méthode de Doss et Sussmann. Comme $x$ n'est en général pas explicite, il est intéressant de définir des schémas d'approximation de cette solution.

2) Intéressons-nous tout d'abord au schéma le plus simple associé à (2), à savoir le schéma d'Euler:

$$
\left\{\begin{array}{l}
\widehat{x}_{0}^{(n)}=x_{0} \\
\widehat{x}_{t}^{(n)}=\widehat{x}_{k / n}^{(n)}+\sigma\left(\widehat{x}_{k / n}^{(n)}\right)\left(g_{t}-g_{k / n}\right)+b\left(\widehat{x}_{k / n}^{(n)}\right)(t-k / n), t \in[k / n,(k+1) / n] .
\end{array}\right.
$$

Théorème 3 Supposons que $\sigma$ soit de classe $\mathrm{C}^{2}$ avec ses deux dérivées bornées et que $b$ soit lipschitzienne. Quand $n \rightarrow+\infty$, on a $\left\|x-\widehat{x}^{(n)}\right\|_{L^{\infty}([0,1])}=O\left(1 / n^{2 \alpha-1}\right)$.

Remarquons que ce théorème n'a un intérêt que si $\alpha>1 / 2$. Lorsque $\alpha \leq 1 / 2$, nous considérons un schéma de type Milshtein d'ordre $m \in \mathbb{N}^{*}$ :

$$
\left\{\begin{aligned}
\check{x}_{0}^{(n)}=x_{0} & \\
\check{x}_{t}^{(n)}=\check{x}_{k / n}^{(n)}+\sum_{j=1}^{2 m} \frac{1}{j !} & P_{j}\left(\sigma, \sigma^{\prime}, \ldots, \sigma^{(j-1)}\right)\left(\check{x}_{k / n}^{(n)}\right)\left(g_{t}-g_{k / n}\right)^{j} \\
& \quad+b\left(\check{x}_{k / n}^{(n)}\right)(t-k / n), t \in[k / n,(k+1) / n] .
\end{aligned}\right.
$$

Les $P_{j}$ sont les fonctions polynomiales données par l'identité formelle suivante:

$$
g^{\prime}=f \circ g \Rightarrow \forall j \in \mathbb{N}^{*}, g^{(j)}=P_{j}\left(f, f^{\prime}, \ldots, f^{(j-1)}\right) \circ g \text { avec } P_{j} \in \mathbb{R}\left[X_{0}, \ldots, X_{j-1}\right] .
$$

Par exemple, nous avons:

$$
\begin{aligned}
& g^{\prime}=f \circ g \Rightarrow P_{1}=X_{0} \in \mathbb{R}\left[X_{0}\right] \\
& g^{\prime \prime}=g^{\prime} \times\left(f^{\prime} \circ g\right)=\left(f f^{\prime}\right) \circ g \Rightarrow P_{2}=X_{0} X_{1} \in \mathbb{R}\left[X_{0}, X_{1}\right], \text { etc. }
\end{aligned}
$$

Théorème 4 Supposons que $\sigma$ soit de classe $\mathrm{C}^{2 m+1}$ avec toutes ses dérivées bornées et que $b$ soit lipschitzienne. Quand $n \rightarrow+\infty$, on $a\left\|x-\check{x}^{(n)}\right\|_{L^{\infty}([0,1])}=O\left(1 / n^{\beta}\right)$ avec $\beta=\inf \{\alpha,(2 m+1) \alpha-1\}$.

Remarque 5 1. En pratique, on choisit l'entier $m$ le plus petit possible tel que $(2 m+$ 1) $\alpha-1 \geq \alpha$ pour obtenir la vitesse optimale $\beta=\alpha$.

2. Le cas $m=1$ (schéma de Milshtein standard) est prouvé dans [6]. Je remercie le referee de cette Note de m'avoir signalé ce travail.

Idées de la preuve du théorème 4. On s'inspire de la démonstration du théorème 2.8 de [6]. Posons $\check{y}_{t}^{(n)}=v\left(g_{t}, \check{x}_{t}^{(n)}\right)$ où $v$ est l'inverse à droite de $u$, c'est-à-dire vérifie $u(x, v(x, y))=y$ pour tous $x, y \in \mathbb{R}$. Grâce à l'hypothèse de régularité sur $\sigma$, on peut effectuer le développement de Taylor de $\check{y}_{(k+1) / n}^{(n)}-\check{y}_{k / n}^{(n)}$ à l'ordre $2 m+1$, autour de $\left(g_{k / n}, \check{x}_{k / n}^{(n)}\right)$. À l'aide de relations algébriques du type de celles contenues dans les lemmes 2.1 et 2.2 de [6], tous les termes dans le développement de Taylor s'annulent et il reste $\check{y}_{(k+1) / n}^{(n)}-\check{y}_{k / n}^{(n)}=O\left(1 / n^{(2 m+1) \alpha}\right)$. En utilisant la définition 4 d'une part et en faisant le 
développement de Taylor d'ordre $2 m+1$ de $\check{x}_{(k+1) / n}^{(n)}-\check{x}_{k / n}^{(n)}$ en utilisant $\check{x}_{t}^{(n)}=u\left(g_{t}, \check{y}_{t}^{(n)}\right)$ d'autre part, nous obtenons

$$
\check{y}_{(k+1) / n}^{(n)}-\check{y}_{k / n}^{(n)}=\mathrm{e}^{-\int_{0}^{g_{k} / n} \sigma^{\prime}\left(u\left(s, \check{y}_{k / n}^{(n)}\right)\right) d s} b \circ u\left(g_{k / n}, \check{y}_{k / n}^{(n)}\right) \frac{1}{n}+O\left(1 / n^{(2 m+1) \alpha}\right) .
$$

En utilisant le contrôle $(2.3 ; 1)$ dans [6], il vient $\sup _{k}\left|\check{y}_{k / n}^{(n)}-y_{k / n}\right|=O\left(1 / n^{\beta}\right)$ puis, comme $u$ est lipschitzienne par rapport à sa deuxième variable: $\sup _{k}\left|\check{x}_{k / n}^{(n)}-x_{k / n}\right|=O\left(1 / n^{\beta}\right)$.

\section{Cas où $g$ est la trajectoire d'un mouvement brownien frac- tionnaire}

De nombreuses applications récentes s'appuient sur une modélisation faisant intervenir des équations différentielles dirigées par un mouvement brownien fractionnaire (en abrégé, mbf): voir par exemple [7], [8] et leurs références. Nous allons étudier le cas où, dans (2), $g$ est la trajectoire d'un mbf $B^{H}$ d'indice $H$ quelconque dans $(0,1)$ :

$$
\left\{\begin{array}{l}
d X_{t}=\sigma\left(X_{t}\right) d B_{t}^{H}+b\left(X_{t}\right) d t, t \in[0,1] \\
X_{0}=x_{0}
\end{array}\right.
$$

Tous les résultat de la théorie générale précédente s'appliquent car il est classique que les trajectoires de $B^{H}$ sont p.s. $\alpha$-höldériennes, pour tout $0<\alpha<H$. Toutefois, dans ce cadre particulier, on peut se poser deux questions naturelles. Premièrement, comment simuler les incréments du mouvement brownien fractionnaire? Un moyen rapide et efficace est d'utiliser la procédure Matlab proposée par Coeurjolly [9] (voir un exemple d'application dans [2], pages 17-18). Deuxièmement, quel est le domaine de convergence (par rapport à $H)$ du schéma de Milshtein d'ordre $m$ associé à (6)? Un élément de réponse est contenu dans la:

Proposition 6 Sous les hypothèses du théorème 4 pour $\sigma$ et b, le schéma de Milshtein d'ordre $m$ associé à (6) converge (resp. ne converge pas) en probabilité vers la solution donnée par la méthode de Doss et Sussmann quand $H>1 /(2 m+1)$ (resp. quand $H \leq$ $1 /(2 m+2))$.

Quand $H \in(1 /(2 m+2), 1 /(2 m+1)]$, on ne sait a priori rien dire sur la convergence du schéma. Toutefois, j'ai de bonnes raisons de penser que le schéma converge bien que cela semble a priori difficile à démontrer. Je renvoie le lecteur intéressé à un article à venir.

Preuve de la proposition 6. La convergence du schéma quand $H>1 /(2 m+1)$ est une application directe du théorème 4 . Supposons maintenant $H \leq 1 /(2 m+2)$ et choisissons $\sigma(x)=x, b(x)=0$ et $x_{0}=1$ dans (4). Un calcul simple montre que

$$
\check{X}_{1}^{(n)}=\prod_{k=0}^{n-1}\left\{1+\sum_{j=1}^{2 m} \frac{1}{j !}\left(B_{(k+1) / n}^{H}-B_{k / n}^{H}\right)^{j}\right\} .
$$

Comme on a, pour $u$ proche de 0 :

$$
1+u+\frac{u^{2}}{2 !}+\ldots+\frac{u^{2 m}}{(2 m) !}=\exp \left(u-\frac{u^{2 m+1}}{(2 m+1) !}+\frac{2 m+1}{(2 m+2) !} u^{2 m+2}+o\left(u^{2 m+2}\right)\right),
$$


on en déduit que

$$
\begin{gathered}
\check{X}_{1}^{(n)}=\exp \left(B_{1}^{H}-\frac{1}{(2 m+1) !} \sum_{k=0}^{n-1}\left(B_{(k+1) / n}^{H}-B_{k / n}^{H}\right)^{2 m+1}+\right. \\
\left.+\frac{2 m+1}{(2 m+2) !} \sum_{k=0}^{n-1}\left(B_{(k+1) / n}^{H}-B_{k / n}^{H}\right)^{2 m+2}+o\left(\sum_{k=0}^{n-1}\left(B_{(k+1) / n}^{H}-B_{k / n}^{H}\right)^{2 m+2}\right)\right) .
\end{gathered}
$$

À l'aide d'une régression linéaire, on peut montrer que, dans $\mathrm{L}^{2}$ et donc en probabilité,

$$
\sum_{k=0}^{n-1}\left(B_{(k+1) / n}^{H}-B_{k / n}^{H}\right)^{2 m+2} \rightarrow\left\{\begin{array}{l}
\frac{(2 m+2) !}{2^{m+1}(m+1) !} \text { si } H=\frac{1}{2 m+2} \\
+\infty \text { si } H<\frac{1}{2 m+2} .
\end{array}\right.
$$

et que $\sum_{k=0}^{n-1}\left(B_{(k+1) / n}^{H}-B_{k / n}^{H}\right)^{2 m+1} \rightarrow 0$ quand $H>1 /(4 m+2)$. Quand $H \leq 1 /(4 m+2)$, en utilisant la même technique que [10], section 5, preuve de 2.b), on peut montrer que $\sum_{k=0}^{n-1}\left(B_{(k+1) / n}^{H}-B_{k / n}^{H}\right)^{2 m+1}$ ne converge pas en probabilité. Donc, en probabilité,

$$
\check{X}_{1}^{(n)} \rightarrow\left\{\begin{array}{l}
\exp \left(B_{1}^{H}+\frac{2 m+1}{2^{m+1}(m+1) !}\right) \neq \exp \left(B_{1}^{H}\right) \text { si } H=\frac{1}{2 m+2} \\
+\infty \text { si } \frac{1}{4 m+2}<H<\frac{1}{2 m+2} \\
\text { ne converge pas si } H \leq \frac{1}{4 m+2} .
\end{array}\right.
$$

Ainsi, comme la solution donnée par la méthode de Doss et Sussmann est $X_{t}=\exp \left(B_{t}^{H}\right)$, le schéma ne converge pas vers elle si $H \leq 1 /(2 m+2)$. Ceci termine la démonstration de la proposition.

Remerciement. Je tiens à exprimer ma reconnaissance envers Pierre Vallois. Ses suggestions et remarques m'ont apporté une aide précieuse.

\section{References}

[1] Young, L. C. An inequality of the Hölder type connected with Stieltjes integration. Acta Math. 67 (1936), 251-282.

[2] Nourdin, I. Calcul stochastique généralisé et applications au mouvement brownien fractionnaire; Estimation non paramétrique de la volatilité et test d'adéquation. Thèse de doctorat (disponible en ligne sur http://www.inourdin.fr.st), Université Nancy I (2004).

[3] Doss, H. Liens entre équations différentielles stochastiques et ordinaires. Ann. IHP 13 (1977) 99-125.

[4] Sussmann, H.J. An interpretation of stochastic differential equations as ordinary differential equations which depend on a sample point. Bull. Amer. Math. Soc. 83 (1977) 296-298.

[5] Karatzas, I., Shreve, S. Brownian motion and stochastic calculus. Springer Verlag, 2nd edition (1991).

[6] Talay, D. Résolution trajectorielle et analyse numérique des équations différentielles stochastiques. Stochastics 9 (1983), 275-306.

[7] Comte, F., Renault, E. Long memory in continuous time volatility models. Math. Finance 8 (1998), 291-323.

[8] Cutland, N., Kopp, P., Willinger, W. Stock price returns and the Joseph effect: a fractional version of the Black-Shole model. Seminar on Stochastic Analysis, Random Fields and Applications, Progress in Probability 36 (1995), 327-351.

[9] Coeurjolly, J-F. Simulation and identification of the fractional Brownian motion: a bibliographical and comparative study. Journal of Statist. Software 5, issue 7 (2000).

[10] Gradinaru, M., Nourdin, I., Russo, F., Vallois, P. m-order integrals and generalized Itô's formula; the case of a fractional Brownian motion with any Hurst index. À paraitre aux Ann. Inst. H. Poincaré Probab. Statist. (2004) 